OPEN ACCESS

Edited by:

Kai G. Kahl,

Hannover Medical School, Germany

Reviewed by:

Samer El Hayek,

University of Miami Health System,

United States

Alessandra Borsini

King's College London,

United Kingdom

*Correspondence:

Lisa S. Olive

lisa.olive@deakin.edu.au

Specialty section: This article was submitted to

Psychosomatic Medicine,

a section of the journal

Frontiers in Psychiatry

Received: 13 September 2021 Accepted: 23 November 2021

Published: 10 February 2022

Citation:

Olive LS, Sciberras E, Berkowitz TS, Hoare E, Telford RM, O'Neil A,

Mikocka-Walus A, Evans S,

Hutchinson D, McGillivray JA, Berk M,

Teague SJ, Wood AG, Olsson C and

Westrupp EM (2022) Child and Parent

Physical Activity, Sleep, and Screen

Time During COVID-19 and

Associations With Mental Health: Implications for Future

Psycho-Cardiological Disease?

Front. Psychiatry 12:774858.

doi: 10.3389/fpsyt.2021.774858

\section{Child and Parent Physical Activity, Sleep, and Screen Time During COVID-19 and Associations With Mental Health: Implications for Future Psycho-Cardiological Disease?}

Lisa S. Olive ${ }^{1,2,3 *}$, Emma Sciberras ${ }^{1,4}$, Tomer S. Berkowitz ${ }^{1}$, Erin Hoare ${ }^{2}$, Rohan M. Telford ${ }^{5}$, Adrienne O'Neil ${ }^{2,6}$, Antonina Mikocka-Walus ${ }^{1}$, Subhadra Evans ${ }^{1}$, Delyse Hutchinson ${ }^{1,4,7}$, Jane A. McGillivray ${ }^{1}$, Michael Berk ${ }^{2,3,8}$, Sam J. Teague ${ }^{1}$, Amanda G. Wood ${ }^{1,4,9}$, Craig Olsson ${ }^{1,4}$ and Elizabeth M. Westrupp ${ }^{1,10,11}$

${ }^{1}$ Centre for Social and Early Emotional Development, School of Psychology, Deakin University, Canberra, VIC, Australia, ${ }^{2}$ Faculty of Health, The Institute for Mental and Physical Health and Clinical Translation (IMPACT), Deakin University, Geelong, VIC, Australia, ${ }^{3}$ Orygen, Elite Sports and Mental Health, and the Department of Psychiatry, The University of Melbourne, Parkville, VIC, Australia, ${ }^{4}$ Murdoch Children's Research Institute, Parkville, VIC, Australia, ${ }^{5}$ Research Institute for Sport and Exercise, University of Canberra, Canberra, VIC, Australia, ${ }^{6}$ The Food and Mood Centre, Deakin University, Geelong, VIC, Australia, ${ }^{7}$ The National Drug and Alcohol Research Centre, University of New South Wales, Sydney, NSW, Australia, ${ }^{8}$ Florey Institute of Neuroscience and Mental Health, The University of Melbourne, Melbourne, VIC, Australia, ${ }^{9}$ College of Health \& Life Sciences, Aston University, Birmingham, United Kingdom, ${ }^{10}$ Department of Paediatrics, University of Melbourne, Melbourne, VIC, Australia, ${ }^{11}$ Judith Lumley Centre, La Trobe University, Melbourne, VIC, Australia

The COVID-19 pandemic has afforded the opportunity for some to improve lifestyle behaviours, while for others it has presented key challenges. Adverse changes in global lifestyle behaviours, including physical activity, sleep, and screen time can affect proximal mental health and in turn distal cardiovascular outcomes. We investigated differences in physical activity, sleep, and screen time in parents and children during early stages of the COVID-19 pandemic in Australia compared to pre-COVID-19 national data; and estimated associations between these movement behaviours with parent and child mental health. Cross-sectional baseline data from the COVID-19 Pandemic Adjustment Study (CPAS; $N=2,365$ ) were compared to nationally representative pre-pandemic data from the Longitudinal Study of Australian Children (LSAC; $N=9$,438). Participants were parents of children aged $\leq 18$ years, residing in Australia. Parents provided self-report measures of mental health, physical activity and sleep quality, and reported on child mental health, physical activity and screen time. Children in CPAS had significantly more sleep problems and more weekend screen time. Their parents had significantly poorer sleep quality, despite increased weekly physical activity. Children's sleep problems were significantly associated with increased mental health problems, after accounting for socioeconomic status, physical activity, and screen time. Poorer parent sleep quality and lower levels of physical activity were significantly associated with poorer mental 
health. Monitoring this cohort over time will be important to examine whether changes in movement behaviour are enduring or naturally improve with the easing of restrictions; and whether these changes have lasting effects on either parent or child mental health, and in turn, future risk for CVD.

Keywords: psychiatry, physical activity, children, adults, depression, anxiety, sleep, screen time

\section{INTRODUCTION}

COVID-19, a disease caused by the Severe Acute Respiratory Syndrome Coronavirus 2 (SARS-Cov-2), was classified as a pandemic by the World Health Organization on March 11, 2020 (1). In an attempt to stop the spread of COVID-19, strict quarantine, lockdown, and social distancing measures were implemented around the world, including in Australia, where Federal and State governments introduced Stage three lockdown (2) in April 2020 to reduce the rate of infection. Measures to prevent the spread of COVID-19, such as home confinement, school closures, restriction on the gathering of groups of people, and the shutdown of community services and facilities (e.g., organised sports, playgrounds, pools, and gyms) are likely to have an impact on a number of important health promoting global movement behaviours [e.g., physical activity, screen time and sleep; $(3,4)]$. These behaviours are closely related to both mental health and risk for cardiovascular disease (CVD). Given that the childhood origins of mental health disorders and CVD are increasingly recognised (5), and there are known links between depression, stress and risk for CVD (6-8), there is potential that adversity associated with the COVID-19 pandemic may have long-term effects on the development of psycho-cardiologic diseases. This is plausible due to the shared risk pathways that have been shown to link a range of mental health indicators (e.g., depression, psychosocial stress, lack of social support and negative emotions) with CVD, including both intermediary markers of CVD risk $(9,10)$ and established CVD [(11); for a review of shared pathways see (12)].

Emerging evidence on how COVID-19 related restrictions may be affecting people's physical activity, sleep quality and screen time has been mixed (13-16) but it is not difficult to see how such restrictions may have a negative impact across these domains. For example, the closure of parks, beaches and sports facilities, a lesser need for active transport as people are urged to stay home and increases in screen use related to working and schooling from home, are likely to reduce the time that children and parents spend in environments associated with physical activity. This may be offset at least in part by physical activity being one of a very small number of permitted activities. Similarly, increased time at home may disrupt normal routines, with increased access to screens and potential changes to bedtime routines. In addition, pre-pandemic research has shown that, on unstructured and non-school days (i.e., similar to COVID-19related home confinement), children are less physically active and more sedentary (17), engage in more recreational screen time (18) and have less regularity in sleep patterns (19). To-date, there has been minimal evidence considering the impact of home confinement on physical activity, sleep and screen time for both children and parents within households, meaning that the overall family burden of changes in movement related behaviours is not well-understood. A family systems perspective on the impact of COVID-19 on movement behaviours may be a novel and conceptual approach, particularly in relation to understanding mental health within families.

Decreased physical activity and increased sedentary behaviour may be a risk factor not only for more proximal indicators of mental health but in turn, for more distal risk of CVD among both children and their parents $(20,21)$. For example, poor sleep is closely linked with symptoms of depression and anxiety in children (22) as well as in adults $(23,24)$. Similarly, lower levels of physical activity and greater amounts of time spent in sedentary activities are linked to poorer mental health outcomes among children, including anxiety and depressive symptoms $(25,26)$ and irritability (27). A similar picture presents itself in adults, where reductions in physical activity are related to increases in mental health symptoms $(28,29)$ and a greater likelihood of a clinical diagnosis, such as major depressive disorder $(30,31)$. Furthermore, any negative change to movement behaviours, brought about by COVID-19 restrictions, may be further compounded by COVID-19 stressors, including educational (school disruption), environmental (e.g., loss of employment, housing) and biopsychosocial (e.g., COVID-19 diagnosis, loss of social support) stressors. Within families, these have the potential to directly impact child health through adverse impacts on parent health. Given that poor mental health is also a known risk factor for CVD, alongside these lifestyle risk factors, gaining a clear understanding of the mental health burden of the COVID-19 pandemic among families and its relationship to global movement behaviours can inform targeted public health efforts for effective prevention and/or intervention.

The aim of the current study was to investigate levels of physical activity, screen time, and sleep during the early stages of COVID-19 social restrictions in Australia, and compare it to pre-COVID-19 national data. Further, we sought to estimate the association between these movement behaviours and a range of mental health indicators among both parents and children. Specific aims were to:

- Compare levels of child screen time, sleep problems and regular bedtime routine during the early stages of the COVID-19 social restrictions to Australian pre-pandemic national data.

- Compare levels of parent sleep quality and physical activity during the early stages of the COVID-19 pandemic to Australian pre-pandemic national data. 
- Investigate the associations between child physical activity, sleep and screen time with child indicators of mental health, including depression symptoms (in children aged 2-18 years), anxiety symptoms (in children aged $2-18$ years) and irritability (in children aged 0-18 years), and whether these associations were moderated by COVID-19 related stressors.

- Investigate the association between parent physical activity and sleep quality with parent depression, anxiety, stress, emotion regulation and positive affect, and whether these associations were moderated by COVID related stressors.

\section{METHODS}

\section{The COVID-19 Pandemic Adjustment Survey (CPAS)}

This study uses cross-sectional baseline data from the COVID19 Pandemic Adjustment Survey (CPAS) (32), which aims to investigate the mental health impacts of the COVID-19 crisis in a sample of Australian parents using regular (longitudinal) online surveys. Participants were recruited via paid and unpaid social media advertisements using a range of methods to increase the representativeness of the sample (e.g., targeting demographic factors/postcodes). Recruitment occurred from 8 to 28th April, 2020, which coincided with the Australian federal and state governments introducing increasingly strict social distancing measures to slow the rate of infection. Participants were 2,365 Australian parents. Parents were eligible to participate if they resided in Australia and were aged $\geq 18$ years, English speaking and a current parent of a child aged $0-18$ years. The current study was approved by the Deakin University Human Ethics Advisory Group (HEAG-H 52_2020).

\section{The Longitudinal Study of Australian Children (LSAC)}

As a means of pre-pandemic comparison, we drew on national data from the Longitudinal Study of Australian Children (LSAC), a nationally-representative prospective cohort study of Australian children and their families (33). Participating children were randomly selected in a two-stage cluster sampling design using Australian postcodes and the Medicare universal healthcare database (34). We used biennial parent-report data (99\% mothers) from three waves of the Baby cohort $(N=5,107)$, covering child ages $0-1$ (Wave 1, collected in 2004), 2-3, and 45 years; and from five waves of the Kindergarten cohort $(4,656)$, covering child ages 6-7 (Wave 2, collected in 2006), 8-9, 10-11, $12-13$, and $14-15$ years.

\section{Measures}

Table 1 provides a summary of the socio-demographic, parent, child and COVID-19 risk measures from CPAS and LSAC used in the current analysis.

\section{Population Weighting}

Post-stratification weights in the CPAS dataset were derived to compensate for differences between the final sample and the national population of parents. Post-stratification weights for CPAS were produced through a raking approach (42), using six demographic factors: (1) geographic location (major city, inner and outer regional areas, and remote areas); (2) child age groups (0-4, 5-9, 10-12, 13-14, and 15-18 years); (3) parent gender (male, female); (4) family structure (single parent, couple family); (5) parent education (did not complete high school, high school completion; and (6) pre-pandemic parent employment status (employed, unemployed). The CPAS dataset was weighted to be equivalent to a subpopulation of Australian adults (i.e., parents of a child 0-18 years), with an estimated total population size of 8.4 million parents. LSAC population weights were also applied in weighted analyses.

\section{Statistical Analysis}

Analyses were conducted in Stata version 16. Item level missing data in the CPAS study ranged from 0 to $8 \%$ on individual variables; and up to $29 \%$ in the LSAC study. Missing data were therefore replaced using multivariate multiple imputation by chained equations for each study separately. All variables from the final analytic models and weights were included in the multiple imputation model to create 50 imputed datasets. All reported results are from the multiply imputed datasets.

To address Aims 1 and 2, proportions and 95\% confidence intervals were calculated for LSAC data using all available timepoints for each measure. Two sets of CPAS estimates were calculated; firstly, for the full available sample; and secondly, for an age-matched subsample consistent with LSAC age ranges to allow comparison with the LSAC national data. LSAC estimates were averaged across available waves within each imputation model, and then combined across the Baby and Kindergarten cohorts using a meta-analysis process based on metaphor in $\mathrm{R}$ $(43,44)$. For CPAS and LSAC, both unweighted and weighted data are presented to ensure reported outcomes are as close to population representation as possible. However, given that our datasets varied from population statistics on several population indicators (see Table 2), we primarily focused on unweighted results. We conducted a series of independent samples $t$-tests to assess unweighted differences between samples/groups.

To address Aims 3 and 4, a series of unadjusted linear regression models, using unweighted CPAS data, were conducted to determine the association between movement behaviours and indicators of mental health in the CPAS sample. Next, adjusted models were conducted to assess the unique contribution of each movement behaviour to each indicator of mental health, while accounting for the contribution of the other concomitant variables in the model. In regression models assessing adult data, adjustments were made for age, gender, deprivation, education, Aboriginal and Torres Strait Islander Peoples status and language other than English (LOTE) spoken at home, and for data related to child factors, adjustments were made for child age, child gender, parent age, parent gender, partner status, deprivation, and parent education. Final adjusted models were derived by including concomitant variables where there was evidence for an unadjusted association with the outcome $(p<0.1)$. Interaction terms were added to assess whether COVID related stressors moderated relationships between the movement behaviours and mental health indicators. 
TABLE 1 | Study measures.

\begin{tabular}{|c|c|}
\hline Construct & Measure (items) \\
\hline emographics & $\begin{array}{l}\text { Parent age, gender, country of birth, Aboriginal and Torres Strait Islander status, whether a language other than English was } \\
\text { spoken at home, highest level of education, relationship status, whether they were living with their partner, number of children in } \\
\text { the household, geographic location and whether they had a mental health or physical health condition. Demographic informatior } \\
\text { prior to COVID-19 included employment and study status, household income, source of income and shortage of money. Parent } \\
\text { also reported on their child's age, gender and whether their child had a neurodevelopmental condition } \\
\text { (attention-deficit/hyperactivity disorder, autism spectrum disorder). }\end{array}$ \\
\hline $\begin{array}{l}\text { COVID-19 } \\
\text { psychological } \\
\text { stressors }\end{array}$ & $\begin{array}{l}4 \text { items adapted from the CoRonavIruS Health Impact Survey (CRISIS) V0.1. (35). Two items asked about participants' feelings } \\
\text { about the likelihood catching COVID-19, and COVID-19 being a serious health risk for the participant (both rated on a } 7 \text {-point } \\
\text { Likert scale ranging from } 1=\text { Strongly Disagree to } 7=\text { Strongly Agree) and two items asked about the extent to which they } \\
\text { experience certain negative feelings (worry, fear) when thinking about their ability to deal with COVID-19 (both items rated on a } \\
\text { 4-point scale from "not at all" to "a great deal"). These items were averaged to from a total score (range, 3-18), with higher } \\
\text { scores reflecting greater COVID-19 related psychological stress ( } \alpha=0.69 \text { ). }\end{array}$ \\
\hline $\begin{array}{l}\text { COVID-19 } \\
\text { environmental } \\
\text { stressors }\end{array}$ & $\begin{array}{l}\text { The following binary coded stressors (presence/absence of stressor) were summed to form a count variable (range, 0-6). } \\
\text { Housing insecurity, financial insecurity; job loss, new job, reduction or increases in work hours, or changes ("redeployment") in } \\
\text { employment; food shortages; and COVID-19 illness (contracting the COVID-19 virus, hospitalisation of themselves or a family } \\
\text { member due to infection, and death of a family member due to the virus). }\end{array}$ \\
\hline
\end{tabular}

Mental health Depression Anxiety Stress Scale (DASS) - Symptoms of depression, anxiety and stress in the last week were assessed using the widely used and well-validated Depression Anxiety Stress Scale-21 [DASS-21; (36-38)]. The scale comprises three 7-item subscales to measure depression ( $\alpha=0.89$ ), anxiety $(\alpha=0.82)$, and stress $(\alpha=0.87)$, with items rated on a 4-point Likert scale ( 0 = Did not apply to me at all to $3=$ Applied to me very much, or most of the time). Higher scores indicate greater symptom severity.

Emotion Difficulties in Emotion Regulation Scale-16 Item Version - Used to assess emotion regulation in parents ( $\alpha=0.95)$. Each item is regulation $\quad$ rated on a 5 -point Likert scale ranging from $1=$ Almost never to $5=$ Almost always (no time frame specified). Higher scores indicate greater emotion regulation difficulties.

Positive affect Positive and Negative Affect Schedule Short Form - A valid and reliable (39) 5-item measure whereby participants rated on a 5-point Likert scale ( $1=$ Very Slightly or Not at All to $5=$ Extremely) whether each affective state applies to them in the present moment $(\alpha=0.80)$. Higher scores indicate greater positive affect.

Physical Parent physical activity was assessed using the LSAC physical activity item, "About how many days each week do you do at activity least 30 min of moderate or vigorous physical activity (like walking briskly, riding a bike, gardening, tennis, swimming, running, etc.?)", rated from 1 to 7 days.

Sleep $\quad$ Parent sleep quality was assessed using the LSAC item, "During the past month, how would you rate your sleep quality overall?" Rated on a 4-point scale from "very good" to "very bad". Item responses were then dichotomised, where response options 1-2 were coded 1 (very good/good) and 3-4 were coded 2 (very bad/bad).

\section{Child measures}

Depression $^{\mathrm{a}} \quad$ Short Mood and Feelings Questionnaire (SMFQ) - 13-item measure of child depression symptoms with items rated on a 3-point Likert scale $(1=$ Not true to $3=$ True) (overall $\alpha=0.87$; children 6 years and above, $\alpha=0.88$; children $<6$ years, $\alpha=0.79$ ). Higher scores indicate greater depression symptoms.

Anxiety ${ }^{a} \quad$ Modified Brief Spence Children's Anxiety Scale - 4-items assessing symptoms of anxiety $(\alpha=0.77)$. Parents rated on a 4-point Likert scale ("never" to "always") how frequently their child had experienced symptoms in the last 2 weeks. Higher scores indicate greater anxiety symptoms.

Irritability $\quad 1$ item, adapted from the CoRonavIruS Health Impact Survey (CRISIS) (35), which asked, "During the past 2 weeks, how irritable or easily angered has your child been?" Parents rated this item on a 5-point Likert scale, ranging from "Not at all" to "Extremely," with higher scores indicating greater child irritability.

Physical Assessed via 1 item, adapted from LSAC, which asked parents, "About how many days each week did your child do at least activity $\quad 30$ min of moderate or vigorous physical activity (like walking briskly, riding a bike, gardening, tennis, swimming, running, etc.?)" Parents provided a rating ranging from 0 to 7 days.

Sleep Sleep pattern was assessed using the LSAC item, "How much is your child's sleeping pattern or habits a problem for you?" rated on a 4-point scale ("not at all a problem" to "a large problem"). Responses dichotomised, where response options 1-2 were coded 1 (not a problem/small problem) and response options 3-4 were coded 2 (moderate problem/large problem) as per previous research $(40,41)$. Child sleep regularity was assessed using the LSAC item, "Does the study child go to bed at regular times?" Parents rated this item on a 5-point scale, ranging from "never" to "always." Item responses were then dichotomised, where response options 1-3 (never/rarely/sometimes) were coded 1 and response options 4-5 (usually/always) were coded 2, with higher scores representing a more regular bedtime.

Screen time Two items from LSAC, which asked how many hours on a typical (item 1) weekday and (item 2) weekend day did their child use media devices at home for non-educational purposes. Each item was rated on a sliding scale from 1 to 24 hours and provided a measure of weekday screen time and weekend day screen time (hours per day).

CPAS, COVID-19 Pandemic Adjustment Survey; LSAC, Longitudinal Study of Australian Children.

a Measured only in children aged $\geq 2$ years of age. 


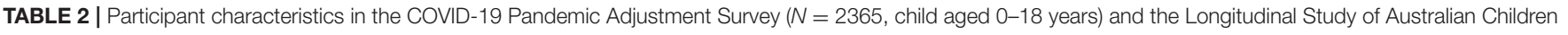
Baby Cohort (Wave 1, $N=5,107$ ) and Kindergarten Cohort (Wave 3, $N=4,331$ ).

\begin{tabular}{|c|c|c|c|}
\hline Participant characteristic ${ }^{a}$ & $\begin{array}{c}\text { CPAS } \\
\text { (Time 1, 2020) }\end{array}$ & $\begin{array}{c}\text { LSAC-B } \\
\text { (Wave 1, 2004) }\end{array}$ & $\begin{array}{c}\text { LSAC-K } \\
\text { (Wave 3, 2008) }\end{array}$ \\
\hline Parent age, m(sd) & $38.30(7.07)$ & $31.0(5.51)$ & $39.1(5.40)$ \\
\hline Child age, m(sd) & $8.66(5.14)$ & & $8.26(0.44)$ \\
\hline \multicolumn{4}{|l|}{ Parent gender } \\
\hline Cisgender men & $19.2 \%$ & $1.5 \%$ & $4.3 \%$ \\
\hline Cisgender women & $80.7 \%$ & $98.6 \%$ & $95.8 \%$ \\
\hline Transgender or non-binary & $<1.00 \%$ & NA & NA \\
\hline \multicolumn{4}{|l|}{ Child gender } \\
\hline Cisgender boy & $50.9 \%$ & $51.1 \%$ & $51.1 \%$ \\
\hline Cisgender girl & $48.7 \%$ & $48.9 \%$ & $49.0 \%$ \\
\hline Transgender or non-binary & $<1.0 \%$ & NA & NA \\
\hline Aboriginal or Torres Strait Islander & $2.1 \%$ & NA & NA \\
\hline Language other than English & $4.5 \%$ & $14.4 \%$ & $13.7 \%$ \\
\hline Parent born overseas & $17.9 \%$ & $18.6 \%$ & $20.4 \%$ \\
\hline Low household income ${ }^{b}$ & $14.0 \%$ & NA & NA \\
\hline Receiving government benefit & $5.8 \%$ & NA & NA \\
\hline Did not complete high school & $9.4 \%$ & $33.3 \%$ & $39.1 \%$ \\
\hline Single parent household & $10.9 \%$ & $9.5 \%$ & $29.3 \%$ \\
\hline \multicolumn{4}{|l|}{ Geographic location } \\
\hline Major cities of Australia & $69.8 \%$ & NA & NA \\
\hline Inner regional Australia & $23.2 \%$ & NA & NA \\
\hline Outer regional Australia & $6.1 \%$ & NA & NA \\
\hline Remote Australia & $1.0 \%$ & $4.3 \%$ & $3.7 \%$ \\
\hline \multicolumn{4}{|l|}{ Number of children } \\
\hline 1 child & $28.4 \%$ & $39.5 \%$ & $8.2 \%$ \\
\hline 2 children & $46.1 \%$ & $36.7 \%$ & $44.2 \%$ \\
\hline 3 children & $18.2 \%$ & $16.1 \%$ & $31.3 \%$ \\
\hline 4 or more children & $5.3 \%$ & $7.6 \%$ & $16.4 \%$ \\
\hline Parent mental or physical health condition & $56.1 \%$ & NA & NA \\
\hline \multicolumn{4}{|l|}{ Child neurodevelopmental condition } \\
\hline Attention-deficit/hyperactivity disorder & $7.3 \%$ & NA & NA \\
\hline Autism spectrum disorder & $8.6 \%$ & NA & NA \\
\hline \multicolumn{4}{|l|}{ COVID-19 related factors } \\
\hline Deprivation index, m(sd) & $0.38(0.95)$ & NA & NA \\
\hline Child home while working & $49.64 \%$ & NA & NA \\
\hline Parent reported CVD & $0.9 \%$ & NA & NA \\
\hline
\end{tabular}

$m(s d)$, Mean (standard deviation), data are unweighted; NA, not applicable.

a Data collected 8th-28th April 2020, data are multiply imputed and thus n cannot be presented for each characteristic.

b $\$ 52,000$ or less per year.

\section{RESULTS}

\section{Participant Characteristics}

Participant characteristics are presented in Table 2. CPAS parents had a mean age of 38 years $(S D=7.07)$, were primarily female $(81 \%)$, resided in a major city $(70 \%)$ and had two children $(46 \%)$ who were on average 9 years old $(S D=5.14)$. Single parent households were a minority $(11 \%)$, and half of the sample had a child at home while working (50\%). Twelve percent of parents reported that their child had an attention-deficit/hyperactivity disorder (ADHD) and/or autism spectrum disorder (ASD) and more than half of parents indicated that they themselves had either a physical or mental health condition. In contrast, LSAC parents in both cohorts were almost all female, but there were similar proportions of LSAC parents born overseas or from single parent families, compared to CPAS parents. A greater proportion of LSAC parents spoke a language other than English and did not complete high school. 
TABLE 3 | Parent and child physical activity, sleep, and screen time compared to Australian normative data (LSAC).

\begin{tabular}{|c|c|c|c|c|c|c|c|c|c|c|c|c|}
\hline & \multicolumn{6}{|c|}{ Unweighted } & \multicolumn{6}{|c|}{ Weighted } \\
\hline & $\%$ & LL & UL & $\%$ & LL & UL & $\%$ & LL & UL & $\%$ & LL & UL \\
\hline \multicolumn{13}{|l|}{ Child sleep problem } \\
\hline Full CPAS sample & 17.7 & 15.9 & 19.4 & & & & 16.4 & 13.6 & 19.2 & & & \\
\hline Full CPAS sample & 20.1 & 18.3 & 21.8 & & & & 21.5 & 18.2 & 24.9 & & & \\
\hline Matched: Children 0-13 years & 13.1 & 11.4 & 14.8 & 2.8 & 2.5 & 3.0 & 15.0 & 11.3 & 18.7 & 3.1 & 2.7 & 3.4 \\
\hline \multicolumn{13}{|l|}{ Parent poor sleep quality } \\
\hline Full sample & 56.0 & 53.9 & 58.1 & & & & 58.3 & 54.4 & 62.2 & & & \\
\hline Matched: Children 0-13 years & 56.7 & 54.3 & 59.0 & 21.0 & 20.4 & 21.6 & 59.1 & 54.6 & 63.5 & 21.6 & 21.0 & 22.3 \\
\hline Full CPAS sample & $3.19(0.07)$ & 3.05 & 3.33 & & & & $3.56(0.13)$ & 3.31 & 3.82 & & & \\
\hline Matched: Children 4-13 years & $2.84(0.07)$ & 2.70 & 2.99 & $2.98(0.01)$ & 2.97 & 2.99 & $3.14(0.13)$ & 2.89 & 3.39 & $3.02(0.01)$ & 3.01 & 3.04 \\
\hline \multicolumn{13}{|l|}{ Screen-time (weekend) } \\
\hline Full CPAS sample & $4.33(0.08)$ & 4.18 & 4.48 & & & & $4.84(0.14)$ & 4.56 & 5.11 & & & \\
\hline Matched: Children 4-13 years & $3.98(0.08)$ & 3.82 & 4.13 & $3.35(0.01)$ & 3.34 & 3.37 & $4.45(0.17)$ & 4.12 & 4.79 & $3.36(0.01)$ & 3.34 & 3.38 \\
\hline \multicolumn{13}{|l|}{ Child physical activity } \\
\hline CPAS: Children $1-18$ years & $4.93(0.05)$ & 4.84 & 5.02 & & & & $4.91(0.08)$ & 4.75 & 5.07 & & & \\
\hline \multicolumn{13}{|l|}{ Parent physical activity } \\
\hline Full CPAS sample & $3.88(0.04)$ & 3.80 & 3.97 & & & & $4.03(0.09)$ & 3.85 & 4.22 & & & \\
\hline Matched: Children 0-13 years & $3.86(0.05)$ & 3.77 & 3.96 & $2.85(0.01)$ & 2.82 & 2.88 & $4.04(0.11)$ & 3.82 & 4.25 & $2.85(0.02)$ & 2.81 & 2.88 \\
\hline
\end{tabular}

"Full CPAS sample" refers to children aged 0-18 years. "Matched" samples refer to CPAS participants that match the child age range available for LSAC normed data. LL/UL refer to the lower limit (LL) and upper limit (UL) estimates from 95\% confidence intervals. se, standard error.

\section{Physical Activity, Screen Time, and Sleep During COVID-19 Compared to National Data}

Table 3 presents parent and child physical activity, sleep, and screen time for CPAS compared to LSAC data.

\section{Child}

During the pandemic, $\sim 1$ in 6 parents reported that their child's sleep was a problem, in both the full CPAS sample (i.e., children $0-18$ years) and the matched CPAS sample (children $0-13$ years). This rate was close to double the rates reported by LSAC parents of children $0-13$ years $\left[17.4\right.$ vs. $8.9 \%, X_{(1,11,621)}^{2}$ $=109.23, p<0.001]$. Although 1 in 5 parents reported their child had no regular bedtime in the full CPAS sample, this was less frequent in the age-matched sample, where approximately 1 in 8 children did not have a regular bedtime. However, this rate was still much higher than the LSAC national data [13.1 vs. $2.8 \%$, $\left.X_{(1,11,621)}^{2}=79.49, p<0.001\right]$.

In the CPAS full sample, children spent on average just over 3 and $4 \mathrm{~h}$ per day, respectively, engaged in weekday, and weekend day recreational screen time. These times were lower in the CPAS age-matched sample (i.e., aged 4-13 years), and there was evidence that CPAS parents reported their child spent less time engaged in recreational screen time on weekdays $\left[t_{(11,183)}\right.$
$=4.31, p<0.001, d=0.12]$ but more time on weekend days $\left[t_{(11619)}=-16.72, p<0.001, d=-0.42\right]$ compared to the LSAC national data.

\section{Parents}

On average, parents participated in at least 30 min of physical activity up to 4 days per week, in both the full and matched CPAS samples, which was higher than the average rates reported in the LSAC national data $\left[t_{(11620)}=-24.96, p<0.001, d=\right.$ $-0.63]$. Over half of parents reported poor sleep quality in both the full and matched CPAS samples, which was more than double the average rates reported in LSAC national data [56.7 vs. $21 \%$, $\left.X_{(1,11,621)}^{2}=1147.12\right]$.

\section{Sensitivity Analysis}

Given the high proportion of CPAS parents who reported having a child with ADHD and/or ASD, and the known links between the movement behaviours and mental health outcomes among these children and parents (45-47), a sensitivity analysis was run removing parents who reported having a child with these conditions to determine the effect on our findings ( $n=$ $15 \%)$. No differences were found in our findings comparing CPAS age-matched sample with and without ADHD/ASD children compared to LSAC national data, indicating this 
TABLE 4 | Associations between child movement behaviours (physical activity, screen time, sleep problems, and sleep routine) and mental health outcomes.

\begin{tabular}{|c|c|c|c|c|c|c|c|c|}
\hline & \multicolumn{4}{|c|}{ Unadjusted } & \multicolumn{4}{|c|}{ Adjusted } \\
\hline & Coefficient & LL & UL & $p$ & Coefficient & LL & UL & $p$ \\
\hline \multicolumn{9}{|l|}{ Child depression $^{a}$} \\
\hline Physical activity & -0.22 & -0.32 & -0.12 & 0.000 & -0.05 & -0.15 & 0.06 & 0.362 \\
\hline Screen time (weekday) & 0.26 & 0.20 & 0.32 & 0.000 & 0.08 & -0.02 & 0.18 & 0.123 \\
\hline Screen time (weekend) & 0.25 & 0.19 & 0.31 & 0.000 & 0.13 & 0.03 & 0.23 & 0.009 \\
\hline Sleep problems & 2.68 & 2.18 & 3.18 & 0.000 & 2.35 & 1.83 & 2.88 & 0.000 \\
\hline No regular bedtime & 1.40 & 0.90 & 1.90 & 0.000 & -0.08 & -0.64 & 0.48 & 0.776 \\
\hline \multicolumn{9}{|l|}{ Child anxiety $^{b}$} \\
\hline Physical activity & 0.03 & -0.03 & 0.09 & 0.298 & 0.05 & -0.01 & 0.11 & 0.090 \\
\hline Screen time (weekday) & 0.02 & -0.02 & 0.05 & 0.382 & -0.02 & -0.07 & 0.03 & 0.455 \\
\hline Screen time (weekend) & 0.02 & -0.02 & 0.05 & 0.312 & 0.05 & -0.01 & 0.10 & 0.078 \\
\hline Sleep problems & 1.40 & 1.11 & 1.68 & 0.000 & 1.36 & 1.06 & 1.65 & 0.000 \\
\hline No regular bedtime & 0.08 & -0.18 & 0.34 & 0.562 & -0.30 & -0.59 & -0.01 & 0.042 \\
\hline \multicolumn{9}{|l|}{ Child Irritability $^{c}$} \\
\hline Physical activity & 0.03 & 0.01 & 0.05 & 0.010 & 0.04 & 0.02 & 0.06 & 0.001 \\
\hline Screen time (weekday) & 0.01 & -0.01 & 0.02 & 0.229 & 0.01 & -0.02 & 0.03 & 0.654 \\
\hline Screen time (weekend) & 0.01 & -0.01 & 0.02 & 0.375 & 0.02 & 0.00 & 0.05 & 0.057 \\
\hline Sleep problems & 0.34 & 0.22 & 0.46 & 0.000 & 0.33 & 0.20 & 0.45 & 0.000 \\
\hline No regular bedtime & 0.01 & -0.10 & 0.13 & 0.809 & -0.01 & -0.14 & 0.12 & 0.862 \\
\hline
\end{tabular}

${ }^{a}$ Adjusted for child age, parent gender, no partner and other movement behaviours.

${ }^{b}$ Adjusted for child age, parent gender, deprivation, no partner, education and other movement behaviours.

${ }^{c}$ Adjusted for child age, parent age, parent gender and other movement behaviours.

over-representation did not change the interpretation of the findings reported.

\section{Association Between Movement Behaviours and Mental Health Outcomes Child}

Unadjusted and adjusted models investigating the association between movement behaviours and mental health outcomes for children in the CPAS cohort are presented in Table 4. In unadjusted models, participating in at least 30 min of moderate or vigorous physical activity per day on fewer days $(B=-0.22,95 \%$ $C I$ [ -0.32 to -0.12 ], $p<0.001)$, spending more hours per day engaged in weekday $(B=0.26,95 \% C I[0.20-0.32], p<0.001)$ and weekend day $(B=0.25,95 \% C I[0.19-0.31], p<0.001)$ recreational screen time, having no regular bedtime $(B=1.40$, $95 \% C I[0.90-1.90], p<0.001)$ and greater sleep problems $(B=$ $2.68,95 \%$ CI [2.18-3.18], $p<0.001)$ were associated with greater depressive symptoms. These associations remained significant in adjusted models for weekend screen time and sleep problems (both $p<0.001$ ).

In unadjusted models, more disrupted sleeping patterns in the CPAS cohort were associated with greater child anxiety symptoms ( $B=1.40,95 \% C I[1.11-1.68], p<0.001)$ but no associations were found for having no bedtime routine, weekday or weekend screen time or physical activity (all $p>0.05$ ). In adjusted models, the association remained for sleep problems $(B$ $=1.30,95 \% C I[1.01-1.60], p<0.001)$ and having no regular bedtime became significantly associated with anxiety symptoms $(B=-0.30,95 \% C I[-0.59$ to -0.01$], p=0.042)$. Higher levels of physical activity $(B=0.04,95 \% C I$ [0.02-0.06], $p=0.001)$ and greater sleep problems $(B=0.33,95 \% C I[0.20-0.45], p$ $<0.001)$ were both associated with child irritability in adjusted models. There was no significant moderating effect of COVID-19 environmental stressors or COVID-19 psychological stressors on the associations between movement behaviours and child mental health outcomes (all $p>0.05$ ).

\section{Sensitivity Analysis}

Given some measures have not yet been validated in a younger sample (e.g., our measure of depression and anxiety have been validated for children as young as 6 years, yet we investigated associations in children as young as 2 years in our full CPAS sample), we undertook a sensitivity analysis, including only children aged 6 years and above. While for the most part our finding remained unchanged, when limiting the sample to 6 years and above, having an irregular bedtime was not associated with anxiety $(p=0.181)$ and weekend screen time was not associated with depression $(p=0.111)$.

\section{Parent}

Unadjusted and adjusted models examining the association between movement behaviours and mental health outcomes for CPAS parents during the pandemic are summarised in Table 5. In adjusted models, lower levels of physical activity and poorer sleep quality were associated with greater parent depressive symptoms. Lower levels of physical activity and poorer sleep quality were also associated with parent anxiety and stress symptoms. Similarly, less physical activity and poorer sleep quality were associated with greater difficulties regulating emotions. More physical 
TABLE 5 | Associations between parent movement behaviours (physical activity and sleep quality) and mental health outcomes.

\begin{tabular}{|c|c|c|c|c|c|c|c|c|}
\hline & \multicolumn{4}{|c|}{ Unadjusted } & \multicolumn{4}{|c|}{ Adjusted } \\
\hline Physical activity & -0.40 & -0.48 & -0.31 & 0.000 & -0.28 & -0.36 & -0.20 & 0.000 \\
\hline Poor sleep quality & 2.63 & 2.29 & 2.98 & 0.000 & 2.16 & 1.82 & 2.51 & 0.000 \\
\hline \multicolumn{9}{|c|}{ Anxiety symptoms ${ }^{b}$} \\
\hline Poor sleep quality & 1.97 & 1.69 & 2.25 & 0.000 & 1.59 & 1.31 & 1.87 & 0.000 \\
\hline \multicolumn{9}{|l|}{ Stress $^{c}$} \\
\hline Physical activity & -0.29 & -0.38 & -0.21 & 0.000 & -0.14 & -0.22 & -0.06 & 0.001 \\
\hline Poor sleep quality & 3.56 & 3.21 & 3.91 & 0.000 & 3.18 & 2.83 & 3.53 & 0.000 \\
\hline \multicolumn{9}{|c|}{ Emotion regulation ${ }^{d}$} \\
\hline Physical activity & -0.89 & -1.13 & -0.65 & 0.000 & -0.56 & -0.80 & -0.33 & 0.000 \\
\hline Poor sleep quality & -1.98 & -2.29 & -1.68 & 0.000 & -1.59 & -1.88 & -1.29 & 0.000 \\
\hline
\end{tabular}

${ }^{a}$ Adjusted for parent age, deprivation, parent education, and other movement behaviours.

${ }^{b}$ Adjusted for parent age, parent gender, deprivation, parent education, parent ATSI status, and other movement behaviours.

${ }^{c}$ Adjusted for parent age, parent gender, deprivation, language other than English spoken at home and other movement behaviours.

${ }^{a}$ Adjusted for parent age, deprivation, parent education, and other movement behaviours.

${ }^{e}$ Adjusted for parent age, parent gender, parent Aboriginal, and Torres Strait Islander Peoples status and other movement behaviours.

activity and greater sleep quality was associated with greater positive affect. COVID-19 environmental stressors moderated the association between sleep quality and depression $(B=0.31$, 95\% CI [0.02-0.60], $p=0.034)$, and moderated the association between sleep quality and difficulties with emotion regulation ( $B=0.96,95 \% C I[0.09-1.84], p=0.031)$, where parents reporting both high COVID-19 stressors and poor sleep quality reported the highest levels of emotion regulation difficulties and depression (see Tables 1, 2). There was no significant moderating effect of COVID-19 psychological stressors on the associations between movement behaviours and parent mental health (all $p>0.05)$.

\section{DISCUSSION}

This study investigated differences in a set of movement behaviours, namely physical activity, sleep and screen time, in parents and children during early stages of the COVID19 pandemic in Australia compared to pre-COVID-19 national data; and estimated associations between these movement behaviours with parent and child mental health. Given the growing body of research establishing the mental health impacts of COVID-19 $(48,49)$, and the known association between movement behaviours with proximal indicators of mental health and more distal risk for CVD, identifying modifiable factors that can be targeted to improve both mental health and risk for CVD, is a priority. Here we show that children and parents under lockdown restrictions report substantially higher rates of sleep problems compared to pre-pandemic rates, with parents also reporting their child had greater levels of recreation screen time on weekends. Children who participated in lower levels of physical activity, had higher levels of recreational screen time and had greater sleep problems also experienced greater depressive symptoms. Greater disruptions to child sleep patterns was also associated with elevated anxiety and irritability symptoms, after accounting for physical activity and screen time. Similarly, poorer parent sleep quality and lower levels of physical activity were associated with poorer parent mental health, increased emotion regulation difficulties, and reduced positive affect. Given the association between the movement behaviours and indicators of mental health for both children and parents during the early stage of COVID-19; coupled with the finding of mostly unfavourable differences in these behaviours compared to pre-pandemic rates, there is potential that adversity associated with the COVID-19 pandemic may have long-term effects on the development of psycho-cardiologic diseases. While longitudinal investigations of this cohort are needed to confirm this hypothesis, at the very least, this study identifies a number of potential modifiable levers that may reduce both risk for more proximal mental health problems and more distal CVD risk.

Australian movement behaviour guidelines recommend that adults be active on most, and preferably all, days of the week, and that children should accumulate $60 \mathrm{~min}$ or more of moderate to vigorous physical activity per day, involving mainly aerobic activities (3). During the pandemic, children were engaging in 5 days of at least $30 \mathrm{~min}$ of moderate or vigorous physical activity, slightly below recommended levels. This finding is somewhat consistent with recent Canadian online survey showing that only $18 \%$ of children were adhering to physical activity recommendations (50). Results are also consistent with Moore et al. who reported in their online survey that children experienced a significant decline in all 
physical activities compared to pre-pandemic rates, with the most dramatic decline seen in outdoor physical activity and sport (16). Whether the lower rates of child physical activity during the early stages of COVID-19 in the current cohort compared to pre-pandemic rates is maintained beyond the initial lockdown restrictions will be important to determine. Physical activity is a known primordial risk factor for later CVD development (51), therefore, any maintenance of low levels of physical activity among our cohort would be of concern. Targeted public health interventions, sympathetic to the COVID-19 context may be required to re-engage children in daily physical activity to achieve pre-pandemic participation rates.

Although parents in the current sample appear to have high levels of physical activity compared to national data, this was still lower than recommended guidelines. Even so, this increase compared to pre-pandemic national data is somewhat heartening, given lower levels of physical activity were associated with poorer mental health across all indicators, even when accounting for sleep quality. Prioritising regular physical activity may be an important strategy for improving parents' mental health, and with COVID-19 related restrictions, it may be that parents are finding more time to engage in physical activity, which may come from greater flexibility in employment conditions or commute time. These higher rates of physical activity among parents are also heartening in terms of the more distal risk for later CVD development, given insufficient levels of physical activity is considered a primordial risk for later CVD (51). Further investigation into the contributing factors that may explain these trends among parents would be worthwhile for public health efforts aiming to improve physical activity levels among the general population, which may in turn improve proximal indicators of mental health indicators but also more distal risk for CVD. However, given the cross-sectional nature of the data, the reverse association cannot be ruled outthat instead, poor mental health contributes to lower physical activity. Regardless of the direction of associations, a key issue however, is that this increase in physical activity did not translate to increases in child physical activity behaviour. Surprisingly, increased physical activity in children was associated with higher levels of irritability. It may be that parents of children with high irritability may be prioritising exercise as an outlet to manage difficult child behaviour.

A growing body of research has demonstrated an increase in sleep problems in the general population as a result of the COVID pandemic (52-56), however, these studies have not systematically compared rates of sleep problems to pre-pandemic levels and/or have not focused on parents and children specifically. The higher levels of child sleep problems and irregular bedtime reported by parents in the CPAS cohort, and the connexion between child sleep problems and indicators of poorer mental health, suggest that sleep has the potential to be an important modifiable factor to improve children's functioning during the COVID-19 pandemic. Consistent with this notion, pre-pandemic studies have connected poorer sleep to increased mental health difficulties in children (57). Even though it may be tempting to relax the rules around children's bedtime during lockdown restrictions, our data suggest that sleep problems during the pandemic continue to be associated with worse mental health in children. Parents in the current study also had substantially higher rates of sleep problems compared to national data, which is consistent with findings from a cross-sectional study of 1,005 adults in France (52). It is notable that over half of parents rated their sleep quality as problematic. Moreover, sleep problems were associated with poorer parent mental health across multiple domains, even when accounting for physical activity levels. This is consistent with the findings of Stanton et al. (55) in their general Australian sample of adults, however, we extend these findings by considering parents specifically, and simultaneously accounting for physical activity levels.

We found mixed evidence concerning screen time. Children had lower levels of recreational screen use during weekdays but higher levels on weekends compared to pre-pandemic national data. It is important to note that parents reported on their child's screen time related to "non-educational purposes." It may be that our findings are affected by variability in the context of schooling across participants. For example, a portion of children are likely to have been on school holidays between late March and late April (varying by State/Territory), overlapping with our data collection period (8th-28th April 2020). Further, about half the sample were juggling work from home while supervising children, and a portion of these children were engaged in remote learning ("home-schooling") at the time of data collection; which is likely to bear an increased screen time load compared to on-campus school learning. It may be that parents restricted non-educational screen time for children already spending much of their day on screens for educational purposes.

In unadjusted analyses, increased weekday and weekend screen time was associated with child depression symptoms but this association was attenuated for weekday screen time when accounting for sleep problems, physical activity and other covariates. Similarly, associations between weekend screen time and child depression symptoms were attenuated when restricting analyses to those aged 6 years and above. Global movement behaviour guidelines for children and adolescents (4) recommend that young children aged 3-4 years engage in no more than $1 \mathrm{~h}$ of sedentary screen time, and that older children and adolescents 5-17 years engage in no more than $2 \mathrm{~h}$ of sedentary recreational screen time. On average, children in our study were engaging in $3 \mathrm{~h}$ of screen time on weekdays and $4 \mathrm{~h}$ on weekends, above recommended guidelines.

It is important to note that children may be using screens to maintain social connexions and this likely has many positive benefits, particularly in the context of limited opportunities for in-person interactions given COVID-19 restrictions. It is also difficult to tease apart the use of screens for educational and noneducational purposes. Overall, the lack of association between screen time and poor child mental health is reassuring for parents. Further, it may not be realistic to reduce child screen time during COVID-19 social distancing restrictions. Instead, family media plans can be used to manage screen time at home and parents should also consider the quality of the content that children are accessing during screen time (58). Fading (graduated extinction) approaches can gradually reduce non-educational screen time to recommended levels. Overall, it will be important 
to monitor screen time in this cohort to see whether screen time naturally reduces once restrictions have lifted.

Surprisingly, we found little evidence that COVID-19 related environmental or psychological stressors moderated associations between movement behaviours and mental health outcomes in children. However, for adults, COVID-19 environmental stressors moderated the association of sleep quality with both depression and emotion regulation. This is consistent with emerging research linking COVID-19 related risk factors to increased sleep difficulties (53). Prevention and intervention efforts that support parents to navigate environmental stressors related to the pandemic need to move beyond helping individuals cope. Focussing on the broader policy levers that may alleviate such stressors, including policies related to financial aid, secure housing and employment, access to COVID-19 safe childcare and school environments, and gendered division of labour will likely have a greater impact on alleviating COVID-19 related stressors and in turn improve parent sleep and mental health. Future research would benefit from focussing on these systemic, policy-based interventions.

Taken together, our findings linking disrupted sleep, low levels of physical activity and weekend screen time to a range of mental health indicators may have important implications for later psycho-cardiological disease. An emerging body of evidence in children and adolescents suggests that early experiences and environments $(59,60)$ in general may influence the course of health and chronic illness. COVID-19 related stressors and the experience of early depression and other emotional distress in children may be particularly relevant to the course of chronic disease development $(60,61)$. A compelling body of evidence has linked both stress and depression to a range of CVD risks and diseases $(8,62-68)$. Understanding the trajectory and prevailing nature of the COVID-19 related stressors and mental health problems reported by parents in the current study has particular importance when considering risk for later CVD. It has previously been suggested that persistent adversity and ongoing chronic stress may result in the greatest risk to CVD and that this increased "wear and tear" on the cardiovascular system over time, induced by stressful experiences that over use and dysregulate stress response pathways that is most problematic $(66,68)$. In addition, better quality sleep and higher levels of physical activity are also associated with a lower risk for developing CVD and better prognosis among those with established CVD $(12,69)$. It is through continuing longitudinal studies such as the CPAS study, and others around the world that the clinical significance of COVID-19 related stressors both in terms of proximal mental health problems and in terms of risk for more distal CVD will be better understood.

Our study has several strengths. The samples are relatively large, and included both children and parents. Our study is comprehensive in that we examine multiple domains of mental health and movement behaviours. We were further able to compare movement behaviours to nationally representative pre-pandemic data. However, several limitations should be considered. First, our sample was recruited online and appears to be more vulnerable than the general population. One example is that the rates of $\mathrm{ADHD}$ and $\mathrm{ASD}$ are much higher than would be expected in the general population. To address this, we applied sample weighting and conducted a sensitivity analysis indicating that our results are robust, both with and without the higher-risk ADHD/ASD children. Second, given that this is a cross-sectional study directionality and causality cannot be inferred and it is possible that the investigated associations are in fact, bidirectional, whereby poor mental health influences sleep, screen time and physical activity. However, population-based longitudinal research has shown that sleep difficulties appear to precede internalising difficulties in childhood, while there is more evidence of a bi-directional association between sleep and behavioural difficulties (57). Longitudinal examination of this cohort will enable further understanding of the directionality between movement behaviours and mental health. Finally, the role other important risk factors for both mental health and CVD risk, including diet and alcohol use, could not be considered as no data was collected on these constructs.

In summary, children in the current study had higher rates of sleep problems and increased weekend screen time compared to national pre-pandemic data. Parents had higher levels of sleep problems compared with pre-pandemic data, while physical activity also appeared to be higher. Poor sleep in both parents and children, and lower levels of physical activity and higher levels of weekend recreational screen time were associated with poorer mental health outcomes. Monitoring this cohort over time will be important to examine whether changes in movement behaviour are enduring or naturally improve with the easing of restrictions and whether these changes have enduring effects on either parent or child mental health, and in turn, more distal risk for psycho-cardiological disease.

\section{CODE AVAILABILITY}

CPAS code is shared on the Open Science Framework.

\section{DATA AVAILABILITY STATEMENT}

Data from CPAS participants who consented to data sharing (91.21\%) will be available through the Australian Data Archive (currently under review) for research projects that are related to CPAS. Please direct enquires to the corresponding author, lisa.olive@deakin.edu.au.

\section{ETHICS STATEMENT}

The studies involving human participants were reviewed and approved by Deakin University Human Ethics Advisory Group (Project No: HEAG-H 52_2020). The patients/participants provided their written informed consent to participate in this study.

\section{AUTHOR CONTRIBUTIONS}

LO conceived the current study, while EW conceived the larger CPAS study. LO, EW, and ES drafted the initial manuscript. EH and $\mathrm{TB}$ were responsible for data management. LO, EW, TB, EH, 
and RT completed the statistical analysis. All authors contributed to and provided feedback on the final draft.

\section{FUNDING}

This work was supported by Deakin University, Centre for Social and Early Emotional Development, School of Psychology, Faculty of Health, Victoria, Australia. LO (1158487) and EH (1156909) are supported by National Health and Medical Research Council (NHMRC) Early Career Fellowships. DH was supported by a

\section{REFERENCES}

1. World Health Organization. Coronavirus Disease (COVID-19) Pandemic: WHO Characterizes COVID-19 as a Pandemic. (2020). World Heal. Organ.

2. Department of Health. Coronavirus (COVID-19) Restrictions [WWW Document]. Aust. Goverment Dep. Heal (2020). Available online at: https:// www.health.gov.au/news/health-alerts/novel-coronavirus-2019-ncovhealth-alert/coronavirus-covid-19-restrictions.

3. Department of Health. Australia's Physical Activity and Sedentary Behaviour Guidelines and the Australian 24-Hour Movement Guidelines [WWW Document]. Aust. Gov. Dep. Heal (2019). Available online at: https://wwwl. health.gov.au/internet/main/publishing.nsf/Content/health-pubhlth-strategphys-act-guidelines.

4. World Health Organization. Guidelines on Physical Activity, Sedentary Behaviour, and Sleep for Children Under 5 Years of Age. (2019). [WWW Document]. World Heal. Organ.

5. Huang RC, Burke V, Newnham JP, Stanley FJ, Kendall GE, Landau LI, et al. Perinatal and childhood origins of cardiovascular disease. Int J Obes. (2007) 31:236-44. doi: 10.1038/sj.ijo.0803394

6. O’Neil A, Fisher AJ, Kibbey KJ, Jacka FN, Kotowicz MA, Williams LJ, et al. Depression is a risk factor for incident coronary heart disease in women: an 18-year longitudinal study. J Affect Disord. (2016) 196:11724. doi: 10.1016/j.jad.2016.02.029

7. De Hert M, Detraux J, Vancampfort D. The intriguing relationship between coronary heart disease and mental disorders. Dialogues Clin Neurosci. (2018) 20:31-40. doi: 10.31887/DCNS.2018.20.1/mdehert

8. Rosengren A, Hawken S, Ounpuu S, Sliwa K, Zubaid M, Almahmeed WA, et al. Association of psychosocial risk factors with risk of acute myocardial infarction in 11119 cases and 13648 controls from 52 countries (the INTERHEART study): case-control study. Lancet. (2004) 364:95362. doi: 10.1016/S0140-6736(04)17019-0

9. Olive LS, Abhayaratna WP, Byrne D, Richardson A, Telford RD. Do self-reported stress and depressive symptoms effect endothelial function in healthy youth? The LOOK longitudinal study. PLoS ONE. (2018) 13:e0196137. doi: 10.1371/journal.pone.0196137

10. Olive LS, Abhayaratna WP, Byrne D, Telford RM, Berk M, Telford RD. Depression, stress and vascular function from childhood to adolescence: a longitudinal investigation. Gen Hosp Psychiatry. (2020) 62:6-12. doi: 10.1016/j.genhosppsych.2019.10.001

11. O'Neil A, Russell JD, Murphy B. How does mental health impact women's heart health? Heart Lung Circ. (2021) 30:59-68. doi: 10.1016/j.hlc.2020.05.111

12. Cohen BE, Edmondson D, Kronish IM. State of the art review: depression, stress, anxiety, cardiovascular disease. Am J Hypertens. (2015) 28:1295302. doi: 10.1093/ajh/hpv047

13. Becker SP, Gregory AM. Editorial perspective: perils and promise for child and adolescent sleep and associated psychopathology during the COVID19 pandemic. J Child Psychol Psychiatry. (2020) 61:757-9. doi: 10.1111/jcpp. 13278

14. Guan H, Okely AD, Aguilar-Farias N, del Pozo Cruz B, Draper CE, El Hamdouchi A, et al. Promoting healthy movement behaviours among children during the COVID-19 pandemic. Lancet Child Adolesc Health. (2020) 4:416-8. doi: 10.1016/S2352-4642(20)30131-0
NHMRC Investigator Grant (1197488). ES was supported by an NHMRC Career Development Fellowship (1110688) and a Veski Inspiring Women's Fellowship. MB was supported by a NHMRC Senior Principal Research Fellowship (1156072).

\section{ACKNOWLEDGMENTS}

The authors thank the participants and Alby Telford for their time given to complete the current study during the COVID-19 pandemic.

15. Cheval B, Sivaramakrishnan H, Maltagliati S, Fessler L, Forestier C, Sarrazin P, et al. Relationships between changes in self-reported physical activity, sedentary behaviour and health during the coronavirus (COVID19) pandemic in France and Switzerland. J Sports Sci. (2021) 39:699704. doi: 10.1080/02640414.2020.1841396

16. Moore SA, Faulkner G, Rhodes RE, Brussoni M, Chulak-Bozzer T, Ferguson LJ, et al. Impact of the COVID-19 virus outbreak on movement and play behaviours of Canadian children and youth: a national survey. Int J Behav Nutr Phys Act. (2020) 17:85. doi: 10.1186/s12966-020-00987-8

17. Sheldrick MP, Maitland C, Mackintosh KA, Rosenberg M, Griffiths LJ, Fry $\mathrm{R}$, et al. Associations between the home physical environment and children's home-based physical activity and sitting. Int J Environ Res Public Health. (2019) 16:4178. doi: 10.3390/ijerph16214178

18. Australian Institute of Family Studies (2016). The Longitudinal Study of Australian Children Annual Statistical report 2015 Melbourne.

19. Olds T, Maher C, Blunden S, Matricciani L. Normative data on the sleep habits of Australian children and adolescents. Sleep. (2010) 33:13818. doi: $10.1093 /$ sleep/33.10.1381

20. Hall G, Laddu DR, Phillips SA, Lavie CJ, Arena R. A tale of two pandemics: how will COVID-19 and global trends in physical inactivity and sedentary behavior affect one another? Prog Cardiovasc Dis. (2020) 64:10810. doi: 10.1016/j.pcad.2020.04.005

21. Pecanha T, Goessler KF, Roschel H, Gualano B. Social isolation during the COVID-19 pandemic can increase physical inactivity and the global burden of cardiovascular disease. Am J Physiol Heart Circ Physiol. (2020) 318:H1441H1446. doi: 10.1152/ajpheart.00268.2020

22. Lovato N, Gradisar M. A meta-analysis and model of the relationship between sleep and depression in adolescents: recommendations for future research and clinical practice. Sleep Med Rev. (2014) 18:5219. doi: 10.1016/j.smrv.2014.03.006

23. Alvaro PK, Roberts RM, Harris JK. A systematic review assessing bidirectionality between sleep disturbances, anxiety, and depression. Sleep. (2013) 36:1059-68. doi: 10.5665/sleep.2810

24. Bao YP, Han Y, Ma J, Wang RJ, Shi L, Wang TY, et al. Cooccurrence and bidirectional prediction of sleep disturbances and depression in older adults: meta-analysis and systematic review. Neurosci Biobehav Rev. (2017) 75:257-73. doi: 10.1016/j.neubiorev.2017.01.032

25. Biddle SJH, Ciaccioni S, Thomas G, Vergeer I. Physical activity and mental health in children and adolescents: an updated review of reviews and an analysis of causality. Psychol Sport Exerc. (2019) 42:146-55. doi: 10.1016/j.psychsport.2018.08.011

26. Rodriguez-Ayllon M, Cadenas-Sánchez C, Estévez-López F, Muñoz NE, Mora-Gonzalez J, Migueles JH, et al. Role of physical activity and sedentary behavior in the mental health of preschoolers, children and adolescents: a systematic review and meta-analysis. Sport Med. (2019) 49:1383-410. doi: 10.1007/s40279-019-01099-5

27. Husárová D, Veselská ZD, Sigmundová D, Madarasová Gecková A. Age and gender differences in prevalence of screen based behaviour, physical activity and health complaints among Slovak school-aged children. Cent Eur J Public Health. (2015) 23:S30-S36. doi: 10.21101/cejph.a4177

28. Schuch FB, Vancampfort D, Firth J, Rosenbaum S, Ward PB, Silva ES, et al. Physical activity and incident depression: a 
meta-analysis of prospective cohort studies. Am J Psychiatry. (2018) 175:631-48. doi: 10.1176/appi.ajp.2018.17111194

29. White RL, Babic MJ, Parker PD, Lubans DR, Astell-Burt T, Lonsdale C. Domain-specific physical activity and mental health: a meta-analysis. Am J Prev Med. (2017) 52:653-66. doi: 10.1016/j.amepre.2016.12.008

30. Schuch F, Vancampfort D, Firth J, Rosenbaum S, Ward P, Reichert T, et al. Physical activity and sedentary behavior in people with major depressive disorder: a systematic review and meta-analysis. J Affect Disord. (2017) 210:139-50. doi: 10.1016/j.jad.2016.10.050

31. Zhai L, Zhang Y, Zhang D. Sedentary behaviour and the risk of depression: a meta-analysis. $\mathrm{Br} J$ Sports Med. (2015) 49:705-9. doi: 10.1136/bjsports-2014-093613

32. Westrupp E, Karantzas G, Macdonald J, Olive L, Youssef GJ, Greenwood C, et al. Study protocol for the COVID-19 Pandemic Adjustment Survey (CPAS): a longitudinal study of Australian parents of a child 0-18 years. Front Psychiatry. (2020) 11:555750. doi: 10.3389/fpsyt.2020.555750

33. Soloff C, Lawrence D, Johnstone R. Sample design (LSAC Technical Paper, No. 1) Melbourne. Aust Inst Fam Stud. (2005).

34. Gray M, Sanson A. Growing up in Australia: longitudinal study of Australian children. Fam Matters. (2005) 72:4-9.

35. Merikangas K, Milham M, Stringari A. The CoRonavIruS Health Impact Survey (CRISIS) 618 V0.1 [WWW Document] (2020).

36. Lovibond P, Lovibond S. Pergamon The structure of negative emotional states: comparison of the Depression Anxiety Stress Scales (DASS) with the Beck Depression and Anxiety. Behav Res Ther. (1995) 33:33543. doi: 10.1016/0005-7967(94)00075-U

37. Antony MM, Cox BJ, Enns MW, Bieling PJ, Swinson RP. Psychometric properties of the 42-item and 21-item versions of the Depression Anxiety Stress Scales in clinical groups and a community sample. Psychol Assess. (1998) 10:176-81. doi: 10.1037/1040-3590.10.2.176

38. Henry JD, Crawford JR. The short-form version of the Depression anxiety stress scales (DASS-21): construct validity and normative data in a large non-clinical sample. Br J Clin Psychol. (2005) 44:22739. doi: 10.1348/014466505X29657

39. Merz EL, Malcarne VL, Roesch SC, Ko CM, Emerson M, Roma VG, et al. Psychometric Properties of Positive and Negative AffectSchedule (PANAS) original and short forms in an AfricanAmerican Community Sample. J Affect Disord. (2013) 151:942-9. doi: 10.1016/j.jad.2013.08.011

40. Quach J, Nguyen C, Connor MO, Wake M. The cumulative effect of health adversities on children's later academic achievement. Acad Pediatr. (2017) 17:706-14. doi: 10.1016/j.acap.2017.03.002

41. Williamson AA, Mindell JA, Hiscock H, Quach J. Longitudinal sleep problem trajectories are associated with multiple impairments in child well-being. $J$ Child Psychol Psychiatry. (2020) 61:1092-103. doi: 10.1111/jcpp.13303

42. Kolenikov S. Calibrating survey data using iterative proportional fitting (Raking). Stata J Promot Commun Stat Stata. (2014) 14:22-59. doi: 10.1177/1536867X1401400104

43. Viechtbauer W. Conducting meta-analyses in R with the metafor Package. J Stat Softw. (2010) 36:1-48. doi: 10.18637/jss.v036.i03

44. Team R, Dc R. A Language and Environment for Statistical Computing. [WWW Document]. R Found. Stat. Comput (2012).

45. Carmassi C, Palagini L, Caruso D, Masci I, Nobili L, Vita A, et al. Systematic review of sleep disturbances and circadian sleep desynchronization in autism spectrum disorder: toward an integrative model of a self-reinforcing loop. Front Psychiatry. (2019) 10:366. doi: 10.3389/fpsyt.2019.00366

46. Jones RA, Downing K, Rinehart NJ, Barnett LM, May T, McGillivray JA, et al. Physical activity, sedentary behavior and their correlates in children with autism spectrum disorder: a systematic review. PLoS ONE. (2017) 12:e0172482. doi: 10.1371/journal.pone.0172482

47. Tong L, Xiong $\mathrm{X}$, Tan $\mathrm{H}$. Attention-deficit/hyperactivity disorder and lifestyle-related behaviors in children. PLoS ONE. (2016) 11:e0163434. doi: 10.1371/journal.pone.0163434

48. Vindegaard N, Eriksen Benros M. COVID-19 pandemic and mental health consequences: systematic review of the current evidence. Brain Behav Immun. (2020) 89:531-42. doi: 10.1016/j.bbi.2020.05.048

49. Nearchou F, Flinn C, Niland R, Subramaniam SS, Hennessy E. Exploring the Impact of COVID-19 on mental health outcomes in children and adolescents: a systematic review. Int J Environ Res Public Health. (2020) 17:8479. doi: 10.3390/ijerph17228479

50. Guerrero MD, Vanderloo LM, Rhodes RE, Faulkner G, Moore SA, Tremblay MS. Canadian children's and youth's adherence to the 24-h movement guidelines during the COVID-19 pandemic: a decision tree analysis. J Sport Health Sci. (2020) 9:313-21. doi: 10.1016/j.jshs.2020.06.005

51. Claas SA, Arnett DK. The role of healthy lifestyle in the primordial prevention of cardiovascular disease. Curr Cardiol Rep. (2016) 18:56. doi: 10.1007/s11886-016-0728-7

52. Beck F, Léger D, Fressard L, Peretti-Watel P, Verger P. Covid-19 health crisis and lockdown associated with high level of sleep complaints and hypnotic uptake at the population level. J Sleep Res. (2020) 30:e13119. doi: 10.1111/jsr.13119

53. Li DJ, Ko NY, Chen YL, Wang PW, Chang YP, Yen CF, et al. Covid-19-related factors associated with sleep disturbance and suicidal thoughts among the taiwanese public: a facebook survey. Int J Environ Res Public Health. (2020) 17:4479. doi: 10.3390/ijerph17124479

54. Lin LY, Wang J, Ou-yang XY, Miao Q. The immediate impact of the 2019 novel coronavirus (COVID-19) outbreak on subjective sleep status. Sleep Med. (2020) 77:348-54. doi: 10.1016/j.sleep.2020.05.018

55. Stanton R, To QG, Khalesi S, Williams SL, Alley SJ, Thwaite TL, et al. Depression, anxiety and stress during COVID-19: associations with changes in physical activity, sleep, tobacco and alcohol use in Australian adults. Int J Environ Res Public Health. (2020) 17:4065. doi: 10.3390/ijerph17114065

56. Jahrami H, BaHammam AS, Bragazzi NL, Saif Z, Faris M, Vitiello MV. Sleep problems during the COVID-19 pandemic by population: a systematic review and meta-analysis. J Clin Sleep Med. (2021) 17:299313. doi: $10.5664 /$ jcsm. 8930

57. Quach J, Cattram N, Williams K, Sciberras E. Bidirectional associations between child sleep problems and internalizing and externalizing difficulties from preschool to early adolescence. JAMA Pediatr. (2018) 172:e174363. doi: 10.1001/jamapediatrics.2017.4363

58. Dalope KA, Woods LJ. Digital media use in families: therories and strategies for intervention. Child Adolesc Psychiatr Clin N Am. (2018) 27:14558. doi: 10.1016/j.chc.2017.11.001

59. Felitti VJ, Anda RF, Nordenberg D, Williamson DF, Spitz AM, Edwards V, et al. Relationship of childhood abuse and household dysfunction to many of the leading causes of death in adults. The Adverse Childhood Experiences (ACE) Study. Am J Prev Med. (1998) 14:245-58. doi: 10.1016/S0749-3797(98)00017-8

60. Miller GE, Chen E, Parker KJ. Psychological stress in childhood and susceptibility to the chronic diseases of aging: moving towards a model of behavioral and biological mechanisms. Psychol Bull. (2011) 137:95997. doi: 10.1037/a0024768

61. Shonkoff JP, Boyce W, McEwen BS. Neuroscience, molecular biology, and the childhood roots of health disparities: building a new framework for health promotion and disease prevention. J Am Med Assoc. (2009) 301:22529. doi: 10.1001/jama.2009.754

62. Bunker SJ, Colquhoun DM, Esler MD, Hickie IB, Hunt D, Jelinek VM, et al. "Stress" and coronary heart disease: psychosocial risk factors. Med J Aust. (2003) 178:272-76. doi: 10.5694/j.1326-5377.2003.tb05193.x

63. Glozier N, Tofler GH, Colquhoun DM, Bunker SJ, Clarke DM, Hare DL, et al. Psychosocial risk factors for coronary heart disease. Med J Aust. (2013) 199:179-80. doi: 10.5694/mja13.10440

64. Frasure-Smith N, Lespérance F. Reflections on depression as a cardiac risk factor. Psychosom Med. (2005) 67:S19S25. doi: 10.1097/01.psy.0000162253.07959.db

65. Frasure-Smith N, Lespérance F. Recent evidence linking coronary heart disease and depression. Can J Psychiatry. (2006) 51:7307. doi: 10.1177/070674370605101202

66. Frasure-Smith N, Lespérance F. Depression and cardiac risk: present status and future directions. Heart. (2010) 96:173-76. doi: 10.1136/hrt.2009.186957

67. Hare DL, Toukhsati SR, Johansson P, Jaarsma T. Depression and cardiovascular disease: a clinical review. Eur Heart J. (2014) 35:136572. doi: 10.1093/eurheartj/eht462

68. Hemingway H, Marmot M. Psychosocial factors in the aetiology and prognosis of coronary heart disease: systematic review of prospective cohort studies. BMJ. (1999) 318:1460-7. doi: 10.1136/bmj.318.7196.1460 
69. Hoevenaar-Blom MP, Spijkerman AMW, Kromhout D, van den Berg JF, Verschuren WM. Sleep duration and sleep quality in relation to 12-year cardiovascular disease incidence: the MORGEN study. Sleep. (2011) 34:1487-92. doi: 10.5665/sleep. 1382

Conflict of Interest: The authors declare that the research was conducted in the absence of any commercial or financial relationships that could be construed as a potential conflict of interest.

Publisher's Note: All claims expressed in this article are solely those of the authors and do not necessarily represent those of their affiliated organizations, or those of the publisher, the editors and the reviewers. Any product that may be evaluated in this article, or claim that may be made by its manufacturer, is not guaranteed or endorsed by the publisher.

Copyright ๑ 2022 Olive, Sciberras, Berkowitz, Hoare, Telford, O’Neil, MikockaWalus, Evans, Hutchinson, McGillivray, Berk, Teague, Wood, Olsson and Westrupp. This is an open-access article distributed under the terms of the Creative Commons Attribution License (CC BY). The use, distribution or reproduction in other forums is permitted, provided the original author(s) and the copyright owner(s) are credited and that the original publication in this journal is cited, in accordance with accepted academic practice. No use, distribution or reproduction is permitted which does not comply with these terms. 